\title{
ON A SIMPLICIAL COMPLEX ASSOCIATED TO THE MONODROMY \\ BY
}

GERALD LEONARD GORDON ${ }^{1}$

\begin{abstract}
Suppose we have a complex analytic family, $V_{t},|t|<1$, such that the generic fibre is a nonsingular complex manifold of complex dimension $n$. Let $T$ denote the monodromy induced from going once around the singular fibre and let $I$ denote the identity map. We shall associate to the singular fibre a simplicial complex $\Gamma$, which is at most $n$-dimensional. Then under certain conditions on the family $V_{t}$ (which are satisfied for the Milnor fibration of an isolated singularity or if the $V_{t}$ are compact Kähler), there is an integer $N>0$ such that $\left(T^{N}-I\right)^{k} H_{k}\left(V_{t}\right)=0$ if and only if $H_{k}(\Gamma)=0$.
\end{abstract}

\section{Introduction.}

1.1. In this article, we let $V$ be a complex hypersurface having normal crossings, where the complex dimension of $V$ is $n$. Then we shall construct a simplicial complex $\Gamma$ corresponding to $V$, where the real dimension of $\Gamma$ is at most $n$. In fact, to each integer $N>0$, we shall associate to $V$ a complex $\Gamma_{N}$, where $\Gamma_{1}=\Gamma$. Then we shall see how these complexes have applications in studying the monodromy about $V$.

To be more specific, suppose we have $V$ embedded in a complex analytic family $V_{t},|t|<1$, where $V_{0}=V$, such that the nearby fibre is nonsingular and let $T$ denote the monodromy induced from going once around the origin. We let $a_{k}$ denote the number of terms of the type

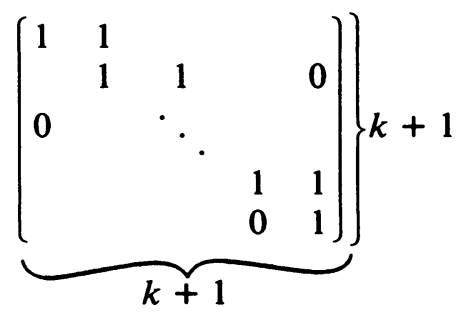

in the Jordan canonical form of the matrix of $T$ acting on $H_{k}\left(V_{t}\right)$. Then if the analytic family is either (i) embeddable in a compact Kähler manifold with $V_{t}$ being compact or (ii) is the Milnor fibration about an isolated singular point, then we shall show that there is an $N$ such that $\operatorname{dim} H^{k}\left(\Gamma_{N}\right)=a_{k}$. Similar results are true for $k=n$ in case the components of $V_{0}$ satisfy certain conditions, regardless of the type of singularity.

Received by the editors July 6, 1978 and, in revised form, April 25, 1979 and September 4, 1979; presented to the Society, March 25, 1978.

AMS (MOS) subject classifications (1970). Primary 14D05, 32C40; Secondary 32 G13.

${ }^{1}$ This work was partially supported by the NSF. 
Throughout this article, we shall always denote $H_{*}(X)$ to mean homology with rational coefficients, for any space $X$. Moreover $H_{*}^{F}(X)$ or $H_{*}^{c}(X)$ will denote closed or compact support, respectively.

The author would like to thank the referee for bringing his attention to the paper by Steenbrink [14] and for providing the present proof of Theorem 3.2.

\section{Construction of the simplicial complex.}

2.1. Let $W$ be a complex manifold of complex dimension $n+1$ and let $V$ be a hypersurface of $W$ having normal crossings, i.e., $V=\cup_{i \in I} X_{i}$ where each $X_{i}$ is a submanifold of $W$ codimension $_{\mathrm{C}}=1$ and $V$ is locally given by $\Pi_{i=1}^{k} z_{i}^{a_{i}}=0$, where $a_{i} \geqslant 1$ is the multiplicity of $X_{i}=\left\{z_{i}=0\right\}$. For $i_{1}, \ldots, i_{k} \in I$ put $X_{i_{1}} \ldots i_{k}=$ $\cap{ }_{j=1}^{k} X_{i_{j}}$. One can suppose that each $X_{i_{1} \ldots i_{k}}$ is connected. If not, take the monoidal transform with center on all but one of the components, which creates the new $X_{i}$ 's but $X_{i_{1} \ldots i_{k}}$ is now connected.

We also suppose that $V$ is connected by looking at each component.

2.2. Then the simplicial complex $\Gamma$ is constructed as follows. To each $i$ with $X_{i} \neq \varnothing$, we associate a vertex $\sigma_{i}$. For $i<j$, we join $\sigma_{i}$ to $\sigma_{j}$ if and only if $X_{i j} \neq \varnothing$. Note that $X_{i j k} \neq \varnothing$ implies that each of the $X_{i j}, X_{j k}$ and $X_{i k}$ is nonempty. Then, we put a 2-simplex $\sigma_{i j k}$ with boundary the $\sigma_{i j}, \sigma_{i k}$ and $\sigma_{j k}$ if and only if $X_{i j k} \neq \varnothing$. We continue in this fashion so that to each $X_{i_{1} \ldots i_{n+1}} \neq \varnothing$ we associate an $n$-dimensional face $\sigma_{i_{1} \ldots i_{n+1}}$ whose boundary will be the sum of the $\sigma_{i_{1} \ldots i_{j} \ldots i_{n+1}}$.

Then $\Gamma$ is a simplicial complex. We formally define the boundary of $\Gamma$ by $\partial \Gamma=\{\tau \mid \tau$ is a face of $\partial \sigma$ for some $\sigma \in \Gamma\}$. If $I$ is finite, then $\Gamma$ is compact. But $\Gamma$ need not be locally finite.

2.3. We note that $\Gamma$ is not necessarily $n$-dimensional. For example, let us start with $\cup_{i \in I} S_{i}$, which is a family of curves in general position. We let $V=$ $\cup_{i \in I}\left(S_{i} \times \mathbf{C} P_{1}\right)$, i.e., $X_{i}=S_{i} \times \mathbf{C} P_{1}$. Then there are no triple points, hence $\Gamma$ is a one-dimensional complex which is isomorphic to the usual dual of $\cup_{i \in I} S_{i}$.

2.4. As stated above, we have a subcomplex of $\Gamma$ which we call its boundary. For example, if $V_{t}$ is defined by $x y z=t$ in $\mathbf{C}^{3}$, then $\Gamma$ will be a two-dimensional triangle.

Thus, in general, we have the exact sequence

$$
0 \rightarrow H_{n}^{c}(\Gamma ; \mathbf{Z}) \rightarrow H_{n}^{c}(\Gamma, \partial \Gamma ; \mathbf{Z}) \stackrel{\partial_{*}}{\rightarrow} H_{n-1}^{c}(\partial \Gamma ; \mathbf{Z})
$$

Recall, $H_{*}^{c}(X)$ denotes homology with compact support.

2.4.1 Definition. Let $B(\Gamma) \subset H_{n}^{c}(\Gamma, \partial \Gamma ; \mathrm{Z})$ be the subgroup defined by $\alpha \in B(\Gamma)$ if and only if $\partial_{*} \alpha$ has a representative of the form $\Sigma \pm \sigma_{i_{1}} \ldots i_{n}$ such that $X_{i_{1} \ldots i_{n}}$ is a noncompact curve.

Then, of course, $H_{n}^{c}(\Gamma ; Z) \subset B(\Gamma)$. The two-simplex of the example $x y z=t$ is a member of $B(\Gamma)$.

2.5. Let $\pi: W \rightarrow D$ be a holomorphic function, where $D$ is the unit disk in the complex $t$-plane. Suppose that if $V_{t}=\pi^{-1}(t)$, then $\pi: W-V_{0} \rightarrow D-\{0\}$ is a locally trivial fibre bundle with fibre a complex manifold and that $V_{0}$ has normal crossings. 
Let $f: D^{\prime} \rightarrow D$ be given by $f(s)=s^{N}$ where $D^{\prime}$ is the unit disk in the complex $s$-plane. Then let $f^{*} W$ be the induced fibre space over $D$. Then Mumford [12] has shown that if $N$ divides the $\operatorname{lcm}\left(a_{1}, \ldots, a_{k}\right)$ and one blows-up along centers over $V_{0}$, then one has a new $V_{0}^{\prime}$ with normal crossings and multiplicities equal to one, i.e., $V_{0}^{\prime}$ is locally given by $\Pi_{i=1}^{k} z_{i}=0$; the $a_{i}$ 's are equal to one.

2.5.1. If $\Gamma$ is the complex which corresponds to $V_{0}$ and we take $D^{\prime} \rightarrow D$ given by $s \rightarrow s^{N}$, we designate by $\Gamma_{N}$ the simplicial complex which corresponds to $V_{0}^{\prime}$, where $V_{0}^{\prime}$ has multiplicities equal to one.

\section{Applications of $\Gamma$ to questions about monodromy.}

3.1. Suppose we have $\pi: W \rightarrow D$ where $\pi^{-1}(0)=V_{0}$ has normal crossings with $\pi \mid W-V_{0}$ being a locally trivial fibre bundle whose fibre $V_{t}$ is a nonsingular manifold. Assume further that $V_{0}$ is locally given by $\Pi_{i=1}^{k} z_{i}=1$, i.e., we study $\Gamma_{N}$ and $V_{0}=\bigcup_{i \in I} X_{i}$. Because $\Gamma_{N}$ is induced from the $N$ to one cover of $D^{\prime} \rightarrow D$, the study of $\Gamma_{N}$ corresponds to the study of $T^{N}$ on the original $W-V_{0}$, which is $T^{\prime}$ on $f^{*} W-V_{0}^{\prime}$.

Then we know from Clemens [3] that $\left(T^{N}-I\right)^{k+1} H_{k}\left(V_{t}\right)=0$. Let $a_{k}$ be the number of terms of the type

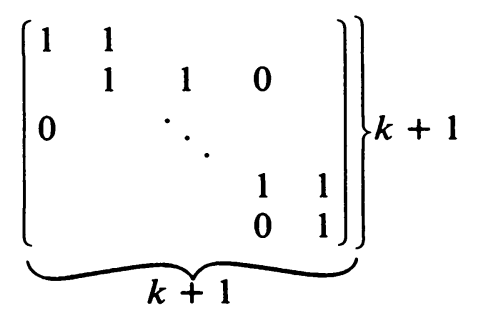

in the Jordan canonical form of the matrix $T^{N}$.

3.2 TheOREM. Suppose that the fibres $V_{t}$ are compact and that there exists an embedding of $W \subset W^{\prime} \times \mathbf{C P} P^{1}$, where $W^{\prime}$ is a compact Kähler manifold. Suppose further that the following diagram commutes.

$$
\begin{array}{cccc}
W & \subset & W^{\prime} \times \mathbf{C} P^{1} \\
\downarrow \pi & & \downarrow \pi^{\prime} \\
D & \subset & \mathbf{C P}
\end{array}
$$

where $\pi^{\prime}$ is the projection onto the second factor. Then $\operatorname{dim} H^{k}\left(\Gamma_{N}\right)=a_{k}$.

Proof of Theorem 3.2. We first prove it for $W^{\prime}$ being complex projective. We use the notation of Steenbrink [14]. We look at the limiting mixed Hodge structure on $H^{k}\left(X_{\infty}\right)$, whose weight filtration we denote by $\left\{W_{i}^{k}\right\}_{i=0}^{n}$. The filtration may be described in two different ways, which are the same by Steenbrink [13, §5]. In one way, $W_{0}^{k}=\operatorname{Im}(T-I)^{k}\left(H_{k}\left(V_{t}\right)\right)$. I the other way there is a spectral sequence converging to $H^{*}\left(X_{\infty}\right)$ with $E_{\infty}^{k, 0}=W_{0}^{k}$ in $H^{k}\left(X_{\infty}\right)$, cf. Steenbrink [14, §2.9]. Furthermore, $E_{2}^{k, 0}=H^{0}\left(\tilde{X}_{i_{1} \ldots j_{k+1}}\right)$ with the differential being the alternating sum of the restriction maps. Here $X_{i_{1} \ldots i_{k+1}}$ denotes the disjoint union of the $X_{i_{1} \ldots i_{k+1}}$. But this cochain complex computes the cohomology of $\Gamma_{N}: E_{2}^{k, 0} \stackrel{\sim}{\rightarrow} H^{k}\left(\Gamma_{N}, C\right)$. The spectral sequence degenerates at $E_{2}$, so that $E^{k, 0} \stackrel{\sim}{\rightarrow} H^{k}\left(\Gamma_{N}, C\right)$. 
However, if $W^{\prime}$ is a compact Kähler manifold, then the techniques still go through. Namely, if $V_{0}$ is a compact Kähler variety, then Hironaka [8] has shown that any complex analytic space can be resolved to normal crossings using monoidal transforms. Furthermore, Blanchard [2] has shown that the monoidal transform of a Kähler variety is still Kähler. Hence we stay in the category of compact Kähler varieties.

Then in this case, $W_{0}^{k}$ is still the image of $(T-I)^{k} H_{k}\left(V_{t}\right)$, cf. Clemens [3]. Moreover, the spectral sequence still has $E_{\infty}^{k, 0}=W_{0}^{k}$ as we are in the case of normal crossings, cf. Griffiths-Schmid [7, Chapter 4]. Furthermore, the spectral sequence still degenerates at $E_{2}$, which follows from the principle of the two types, cf. Gordon [6]. Q.E.D. for Theorem 3.2

3.3. Suppose $V_{0}$ dominates a hypersurface $\tilde{V} \subset \mathrm{C}^{n+1}$ for $n \geqslant 1$ and suppose that $\tilde{V}$ has an isolated singularity at the origin. Let $X_{0}$ denote the "inverse" image of the proper transform of $\tilde{V}$ in $X_{0}$. I.e., because the multiplicity of the proper transform of $\tilde{V}$ in any resolution is always one, there is only one component of $V_{0}$ which maps onto $\tilde{V}$. All the other components are sent to the isolated singularity. Let $X_{0}$ denote this component.

We let $\Gamma_{N}^{\prime} \subset \Gamma_{N}$ be the simplicial complex associated to $\cup_{i>0} X_{i}$. This corresponds to removing the open star of the vertex $\sigma_{0} \in \Gamma_{N}$.

Let $\tilde{V}$ be defined by $\{f=0\}$. Recall that the Milnor fibre associated to $\tilde{V}$ is given by $V_{t}=\left\{z \in \mathbf{C}^{n+1} \mid f(z)=t\right.$ and $\left.|z|<\varepsilon\right\}$ for $\varepsilon$ sufficiently small, $0<|t| \ll \varepsilon$.

3.4 THEOREM. If we are in the situation of 3.3 , then $\operatorname{dim} H^{k}\left(\Gamma_{N}^{\prime}\right)=a_{k}$.

Proof of Theorem 3.4. This follows from Steenbrink [14, Corollaries 3.9 and 3.10]. Namely to define the mixed Hodge structure on the Milnor fibre, one is forced to mod out by the contribution given by the proper transform. This corresponds to removing the star of the vertex $\sigma_{0}$ in $\Gamma_{N}$. Q.E.D. for Theorem 3.4

3.5. We note that one must pass to $\Gamma_{N}^{\prime}$ as seen in the example in 4.2 that follows.

3.6 Definition. A complex manifold $X$ is quasi-Kähler if there is a compact Kähler manifold $\bar{X}$ such that $X$ embeds in $\bar{X}$ so that $\bar{X}-X$ is a subvariety (perhaps empty).

3.7 THEOREM. 3.7.1. Without any restrictions on $V_{t}$, we have

(i) if we take homology with closed support on $V_{t}$, then $\operatorname{dim} H_{c}^{n}\left(\Gamma_{N}\right) \geqslant a_{n}$,

(ii) if we take homology with compact support on $V_{t}$, then $\operatorname{dim} B\left(\Gamma_{N}\right) \geqslant a_{n}$.

3.7.2. If each of the $X_{i_{1}} \ldots i_{r}$ is quasi-Kähler and we take homology with closed support on $V_{t}$, then $\operatorname{dim} H_{c}^{n}\left(\Gamma_{N}\right)=a_{n}$.

3.7.3. If each of the $X_{i_{1}} \ldots i_{r}$ is quasi-Kähler and in addition $V_{0}$ dominates an irreducible variety, then in homology with compact support we have $\operatorname{dim} B\left(\Gamma_{N}\right)=a_{n}$.

Proof of Theorem 3.7. 3.7.1 follows from the Leray spectral sequence associated to the inclusion of $W-V_{0} \subset W$. The reason we must distinguish between compact and closed support is discussed in Gordon [5, §4].

3.7.2 follows because in Gordon [6] it is shown that under these hypotheses, the Leray spectral sequence degenerates at $E_{2}$. 
To prove 3.7.3, if $V_{0}$ dominates an irreducible variety we let $X_{0}$ denote the proper transform. For $n>2$, each curve $X_{i_{1}} \ldots i_{n}$ will be compact if $i_{j}>0$ for all $j$. This is because each curve will be of codimension greater than one. Hence for $\boldsymbol{n}>2$, $B\left(\Gamma_{N}\right)=H_{n}^{c}\left(\Gamma_{N}\right)$, as each closed $n$-simplex of $\Gamma_{N}$ must have on its boundary at least one $\sigma_{i_{1}} \ldots i_{n}$ with $i_{j}>0$ for all $j$. Q.E.D. for Theorem 3.7

3.8. We need the hypothesis that $V_{0}$ dominates an irreducible variety, for if one

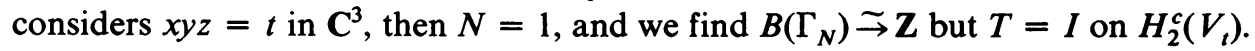

3.9. What Theorem 3.7 says is that to compute the space Image $\left(T^{N}-I\right)^{n} H_{n}\left(V_{t}\right)$, each $n$-tuple point of $V_{0}$ corresponds to a generator and the relations are given by the curves $X_{i_{1}} \ldots \hat{j}_{j} \ldots i_{n}$ in closed support (but only the compact curves in compact support). Hence in dualizing to form $\Gamma_{N}$, each $n$-face of $\Gamma_{N}$ is a generator, and the $(n-1)$-faces give relations; but to compute compact support, we allow relative cycles whose boundaries correspond to compact curves.

3.10. It would be nice to say that if each of the $X_{i_{1} \ldots i}$ is quasi-Kähler, then if $H^{k}\left(\Gamma_{N}\right) \neq 0$, we have that $\left(T^{N}-I\right)^{k} H_{k}\left(V_{t}\right) \neq 0$, as in Theorem 3.2. However, this is not so. For example, let us consider the example of Kodaira [10] of a family of Hopf surfaces $V_{t}$ such that the singular fibre $V_{0}$ is a surface acquiring a double curve. Here $V_{t}$ is diffeomorphic to $S^{1} \times S^{3}$. If we blow-up along this double curve, we obtain $X_{0}$ and $X_{1}$, nonsingular compact Kähler surfaces with $X_{0} \cap X_{1}$ being two disjoint lines. Then blow-up along one of these lines obtaining $X_{2}$, so that $X_{i} \cap X_{j}$ is a line for $i \neq j$ and there are no triple points. Also, the multiplicity of $X_{1}$ is 2 and $X_{2}$ is 3. Thus $N=6$. Then $\Gamma$ is the boundary of a two-simplex, so that $H^{2}\left(\Gamma_{6}\right)=0$ but $H^{1}\left(\Gamma_{6} ; \mathrm{Z}\right) \stackrel{\sim}{\rightarrow} \mathrm{Z}$. But $T^{6}=I$ on $H_{2}\left(V_{t}\right)$. (In fact, $T^{2}=I$.)

What happens is that if one forms the 1-cycle in $V_{0}$ by joining a point in $X_{i} \cap X_{j}$ by a real line $c_{i}$ in $X_{i}$ to $X_{i} \cap X_{k}$ for $\{i, j, k\}=\{0,1,2\}$, then as $z=t$ defines $V_{t}$ near $X_{i}$, we can find a section over $c_{i}$ in $V_{t}$, hence we get a one-cycle $\alpha_{1}$ in $V_{t}$. Then $\left[\alpha_{1}\right] \neq 0$, since $0 \neq\left[c_{0}+c_{1}+c_{2}\right] \in H_{1}\left(V_{0}\right)$. Hence $\alpha_{1}$ is a representative of the generator of $H_{1}\left(V_{t}\right)$. Let $a_{i j}$ be the class which represents the one-cycle in $V_{t}$ which is the fibre over a point in $X_{i j}$, cf. Gordon [5, 2.4] where $a_{i j}=g_{*}^{-1}\left(\gamma_{0, i j}\right)$ in the notation of [5]. Then $\left(T^{6}-I\right)\left[\alpha_{1}\right]=a_{10}+a_{02}+a_{12}$. But in this example, $a_{12}=$ $a_{10}, a_{12}=a_{20}$, and also $a_{10}=2 a_{20}$. The last equality follows because $X_{01}$ is not homologous to $X_{02}$ in $X_{0}$. Hence, $a_{i j}=0$, i.e., $\left(T^{6}-I\right)\left[\alpha_{1}\right]=0$.

Thus, $H_{k}\left(\Gamma_{N} ; Z\right) \neq 0$ implies one can find an $\alpha \in H_{k}\left(V_{t}\right)$ with $\left(T^{N}-I\right)^{k} \alpha=\alpha^{\prime}$, but we do not a priori know if $\alpha^{\prime}$ is a nonzero class of $H_{k}\left(V_{t}\right)$.

3.11. Given an element of $H^{k}\left(\Gamma_{N}\right)$, one can easily construct the element $\alpha \in$ $H_{k}\left(V_{t}\right)$ with $\left(T^{N}-I\right)^{k} \alpha \neq 0$. The construction is the same as is done in Gordon [5, $\S \S 4$ and 5], especially [5, Propositions 4.4.1 and 5.3]. An example is given in 4.1.2.

\section{Some examples.}

4.1. In this section, we shall give some examples of the simplicial complex. We shall first give an example of an isolated singularity for which $H_{n}\left(\Gamma_{N}\right) \neq 0$. Let us take the example of Malgrange [11].

4.1.1 Proposition. Let $\tilde{V} \subset \mathbf{C}^{3}$ be defined by $x^{2} y^{2} z^{2}+x^{8}+y^{8}+z^{8}=0$. Then there is a resolution of $\tilde{V}$ to $V_{0}$ such that the second betti number of $\Gamma$ is 4 . 
Proof of Proposition 4.1.1. We shall always denote the proper transform by $X_{0}$.

First take the monoidal transform with center the origin in $\mathbf{C}^{3}$. Then $X_{1} \cap X_{0}$ gives 3 lines each counted twice, and we blow-up along each of these lines yielding $X_{2}, X_{3}$ and $X_{4}$. This gives a resolution of $\tilde{V}$ with $X_{1} \cap X_{0}=\varnothing$. For $2<i<j<4$ we have that $X_{i} \cap X_{j} \cap X_{0}$ has two distinct points.

But to construct $\Gamma$, it is necessary that each component of $X_{i j k}$ be connected, so we blow-up one of the two triple points for each of the $X_{i j 0}$. This gives us $X_{5}, X_{6}$ and $X_{7}$ with the multiplicity of $X_{i}$ for $i=0,1,2,3,4,5,6,7$ being $1,6,8,8,8,2,2,2$, respectively.

Then with all this data, we can give a presentation of $\Gamma$ : there are 8 vertices, 18 edges, and 15 faces. Then $\Gamma$ can be described as: We start with three one chains, $\sigma_{23}, \sigma_{34}$ and $\sigma_{24}$, which form a one-cycle $\gamma_{1}$. Then we form two cones over $\gamma_{1}$ with vertices $\sigma_{0}$ and $\sigma_{1}$. This defines one 2-cycle. Over each of the boundaries of the 2-dim faces $\sigma_{023}, \sigma_{034}$ and $\sigma_{024}$, we form the cones with vertices $\sigma_{5}, \sigma_{6}$ and $\sigma_{7}$ respectively. This gives 3 other 2 -cycles and finishes the description of $\Gamma$. Q.E.D. for Proposition 4.1.1

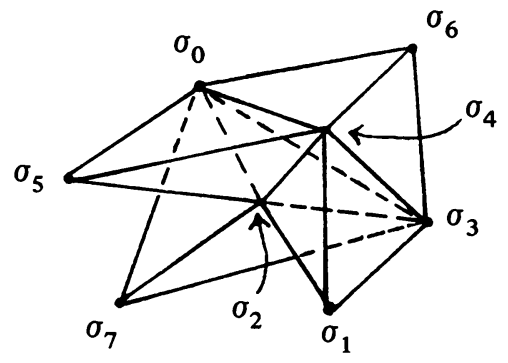

Figure 1

4.1.2. One can easily give a class $\gamma_{2} \in H_{2}\left(V_{t}\right)$ with $\left(T^{24}-I\right)^{2} \gamma_{2} \neq 0$ where $V_{t}=\left\{(x, y, z) \mid x^{2} y^{2} z^{2}+x^{8}+y^{8}+z^{8}=t\right\}$. Namely, take the cone with vertex $\sigma_{7}$.

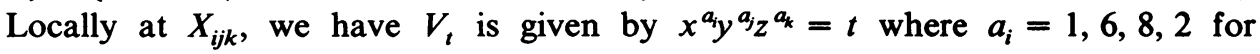
$i=0,2,4,7$, respectively. We note that if $P$ is the triple point, then $\left[\tau_{1, i} \tau_{2, j} \tau_{3, k}(P)\right]$

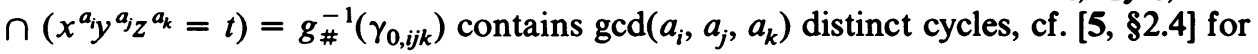
the notation being used.

Hence, take the map of the unit disk into itself given by $s \rightarrow s^{24}=t$ where $24=\operatorname{lcm}(1,2,6,8)$. Then if we take the induced fibre product from this mapping, we find that the number of points mapping onto $X_{i j k}$ is $\operatorname{gcd}\left(a_{i}, a_{j}, a_{k}\right)$, hence we get one point for $X_{0 j k}$ and two points for $X_{247}$. Similarly, $X_{47}$ and $X_{27}$ are covered two to one, so that we must add a new component $X_{8} \approx X_{7} \approx \mathbf{C} P_{2}$ with $X_{07}=X_{08} \approx$ $\mathrm{C} P_{1}$ and $X_{28} \approx X_{48} \approx \mathrm{C} P_{1}$ and $X_{248} \neq \varnothing$. Hence when we blow-up along $X_{08}$ to get normal crossings, we get an $X_{9}$ and the following configuration as a part of $\Gamma_{24}$. 


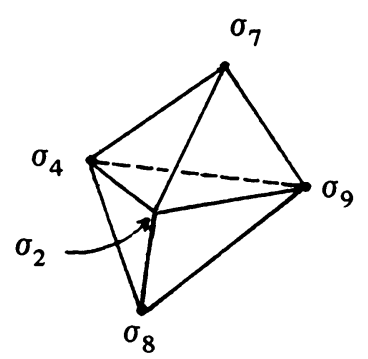

Figure 2

In each double curve, $X_{i j}$, where $(i, j)=\{(2,4),(2,7),(2,9),(8,9),(4,8),(2,8)$, $(4,9),(7,9),(4,7)\}$, take a real line $c_{i j}$ between $X_{i j k}$ and $X_{i j l}$ where the triples run over 247, 248, 489, 289, 479 and 279. Then let $\gamma_{i}=\Sigma_{j} c_{i j}$, which is a one-dimensional cycle in $X_{i}$, for $i=2,4,7,8,9$. Then $\gamma_{i}=\partial_{\#} c_{i}$ as noted in Gordon [5, §5]. Let $\tilde{c}_{i}$ be the cross-section in $V_{t}$ over $c_{i}$, as discussed in [5, Proposition 5.3], and let $\alpha_{1}=\Sigma_{i} \tilde{c}_{i}$. Then $\left(T^{24}-I\right)^{2} \alpha_{1} \neq 0$.

4.2. That we must pass to $\Gamma_{N}^{\prime} \subset \Gamma_{N}$ for isolated singularities is given by the following example. Consider the singularity $\tilde{V}=\left\{\left(x^{2}+y^{3}\right)\left(x^{3}+y^{2}\right)+z^{2}=0\right\}$ in $C^{3}$. Then $\tilde{V}$ is nonsingular except at 6 isolated points.

4.2.1 Proposition. There is a resolution of $\tilde{V}$ to $V_{0}$ such that the second betti number of $\Gamma$ associated to $V_{0}$ is equal to one.

Proof of Proposition 4.2.1. We shall just briefly sketch the resolution. First blow-up the origin in $\mathbf{C}^{3}$. Then this creates $X_{1}$ with $X_{1} \cap X_{0}$ a line in $\mathrm{CP}_{2}$ such that $X_{0}$ is singular along this line. Then blow-up this line creating $X_{2}$ with $X_{2} \cap X_{0}$ being two lines meeting in two points $P_{1}$ and $P_{2}$. Next, blow-up each of the points $P_{1}$ and $P_{2}$ creating $X_{3}$ and $X_{4}$ such that $X_{i} \cap X_{0}=X_{i} \cap X_{2}=$ a line for $i=3$ and 4. Then blow-up along $X_{i} \cap X_{0}$ for $i=3$ and 4 which creates $X_{5}$ and $X_{6}$, respectively. Finally, we blow-up along one of the lines of $X_{2} \cap X_{0}$, yielding an $X_{7}$, and $X_{0}$ is nonsingular in a neighborhood of $\cup_{i} X_{i} \cap X_{0}$.

Hence, we have a resolution of the origin with eight components $X_{i}, i=$ $0,1, \ldots, 7$, of multiplicity $(1,2,4,5,5,10,10,5)$ respectively. There are 8 vertices, 12 edges and 6 faces on $\Gamma$.

The other five points are given by a 2-fold covering branched along 2 curves intersecting transversely at smooth points of each of the curves, i.e., $0=z^{2}+$ $\left(x+g_{1}(x, y)\right)\left(y+g_{2}(x, y)\right)$ is the local equation of $\tilde{V}$ at each of the other 5 isolated points, where the leading term of the $g_{i}$ is of degree two. Hence, each of the five singular points will be resolved with one monoidal transform which contributes $X_{8}, X_{9}, X_{10}, X_{11}, X_{12}$, all of multiplicity two.

A representation of $\Gamma$ is given in Figure 3. Q.E.D. for Proposition 4.2.1 


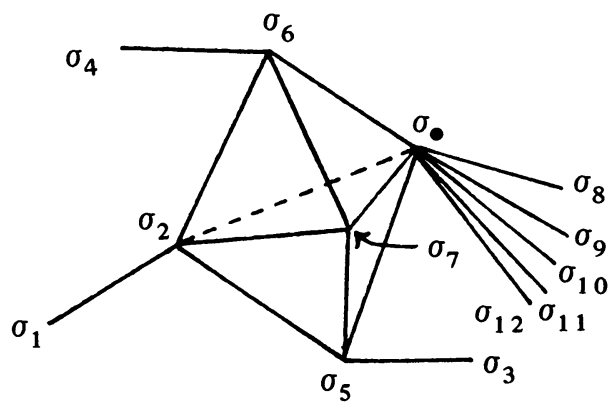

Figure 3

Thus, if $V_{t}=\left\{\left(x^{2}+y^{3}\right)\left(x^{3}+y^{2}\right)+z^{2}=t\right\}$, then for $t$ sufficiently small, and for all $N,\left(T^{N}-I\right)^{2} H_{2}^{c}\left(V_{t}\right) \neq 0$. This follows because if for some $N$ this were not true, i.e., $\left(T^{N}-I\right)^{2} H_{2}^{c}\left(V_{t}\right)=0$, it would also be zero if we replace $N$ by (20) $\cdot N$. But by Proposition 4.2.1 and Theorem 3.2, $\left(T^{20-N}-I\right)^{2} H_{2}^{c}\left(V_{t}\right) \neq 0$.

But, if we consider the local monodromy, i.e., $V_{t} \cap D_{e}$, for $D_{e}$ a sufficiently small ball about the origin, we have that $\left(T^{20}-I\right)^{2} H_{2}\left(V_{t} \cap D_{\varepsilon}\right)=0$. This last fact follows from the results of Sebastiani-Thom [12] which state that the monodromy of $V_{t} \cap D_{\varepsilon}$ is minus the monodromy of $\left(\left(x^{2}+y^{3}\right)\left(x^{3}+y^{2}\right)=t\right) \cap D_{\varepsilon}$.

The reason this happens is as follows: At all the triple points $x^{a_{1}} y^{a_{2} a_{3}}=t$, we have $\operatorname{gcd}\left(a_{1}, a_{2}, a_{3}\right)=1$, so that when we form the 20 to one cover, $20=$ $\operatorname{lcm}(1,2,4,5,10)$, we add no new triple points, hence no new faces are added when we form $\Gamma_{20}$.

However, if one forms the one-cycle $\gamma_{0}$ in $X_{0}$ by joining $X_{026}$ to $X_{067}$ by a real line in $X_{06}$, joining $X_{067}$ to $X_{057}$ by a real line in $X_{07}, X_{057}$ to $X_{025}$, and finally, $X_{025}$ back to $X_{026}$, then $\gamma_{0}=\partial_{\#} c_{0}$. Furthermore, $c_{0}$ is a compact 2-chain in $X_{0}$ such that if $\pi$ : $V \rightarrow \tilde{V}$ is the resolution, then $\pi_{\#} c_{0}$ is a compact 2-chain in $\tilde{V}-S$, where $S$ is the 6 points which constitute the singular locus of $\tilde{V}$. But $\pi_{\#} c_{0}$ cannot be deformed so that it lies in $D_{\varepsilon} \cap X_{0}$.

However, as in 4.1.2, we can form $\alpha_{2} \in H_{2}\left(V_{t}\right)$ and $\left(T^{20}-I\right) \alpha_{2}=\alpha \in H_{2}\left(V_{t}\right)$ such that $\alpha_{2}$ has a representative which lies in $V_{t} \cap D_{\varepsilon}$, i.e., $\alpha_{2} \in H_{2}\left(V_{t} \cap D_{\varepsilon}\right)$ and $T^{N} \alpha_{2} \neq \alpha_{2}$ for all $N$.

Analogous to this, one can show

4.2.2 Proposition. Suppose $P$ is an isolated singularity of a hypersurface in $\mathbf{C}^{n+1}$ and there is a resolution of the isolated singularity so that $H^{n}\left(\Gamma_{N}\right) \neq 0$ for some $N$. Then for all $M,\left(T^{M}-I\right)^{n-1} \neq 0$, where $T$ denotes the local monodromy about $P$.

4.3. One can also give examples with $n>1$ where $\left(T^{N}-I\right)^{2} \neq 0$, but $H_{n}(\Gamma)=$ 0 , i.e., one must go to $\Gamma_{N}$ for $H_{n}\left(\Gamma_{N}\right) \neq 0$. For example, take the example of Karras given by $z^{3}=\left(x+y^{6}\right)\left(x^{2}+y^{6}\right)$. Then the monodromy of the Milnor fibre has $\left(T^{990}-I\right)^{2}=0$ and $H^{2}(\Gamma)=0$. But $H^{2}\left(\Gamma_{990}\right) \neq 0$, which means that if one looks at the monodromy in all of $C^{3},\left(T^{990}-I\right)^{2} \neq 0$. 
4.4. One can give a large class of examples where $H^{n}\left(\Gamma_{N}\right) \neq 0$. Namely, let $V\left(n_{0}, \ldots, n_{n} ; m_{0}, \ldots, m_{n}\right)=\left\{\left(z_{0}, \ldots, z_{n}\right) \mid \prod_{i=0}^{n} z_{i}^{n_{i}}+\sum_{i=0}^{n} z_{i}^{m_{i}}=0\right\}$ with each $m_{i}$ $>1$ and each $n_{i}>0$. Then by the same method used by Malgrange [11], one can show that if all the $n_{i}$ and $m_{i}$ are even, then there is an $N>0$ such that for the monodromy of the Milnor fibre,

$$
\left(T^{N}-I\right)^{n} \neq 0 \text { if and only if } \sum_{i=0}^{n} \frac{n_{i}}{m_{i}}<1 .
$$

After having made the calculation for several other examples, it appears that $H^{n}\left(\Gamma_{N}\left(n_{0}, \ldots, n_{n} ; m_{0}, \ldots, m_{n}\right)\right) \neq 0$ when $\sum_{i=0}^{n} n_{i} / m_{i}<1$, even if the $n_{i}$ or $m_{i}$ are odd.

There seems to be a connection between these examples and with the concept of modality of Arnol'd [1]. For example, $x^{p}+y^{q}+z^{r}+x y z$ is unimodal if $1 / p+$ $1 / q+1 / r<1$. For all of these, we have that $H^{2}\left(\Gamma_{N}(1,1,1 ; p, q, r)\right) \neq 0$ if $1 / p+1 / q+1 / r<1$. However, $x^{3}+y^{3}+z^{4}+x y z^{2}$ is also unimodal (it is $U_{12}$ in [1, p. 227]), but $T^{24}=I$ for this singularity.

\section{BIBLIOGRAPHY}

1. V. I. Arnol'd, Critical points of smooth function and their normal forms, Russian Math. Surveys 30 (1975), 1-75.

2. A. Blanchard, Sur les variétés analytiques complexes, Ann. Ecole Norm. Sup. (3) 73 (1956), 157-202.

3. C. H. Clemens, Picard-Lefshetz theorem for families of nonsingular algebraic varieties acquiring ordinary singularities, Trans. Amer. Math. Soc. 136 (1969), 93-108.

4. __ Degeneration of Kähler manifolds, Duke Math. J. 44 (2) (1977), 215-290.

5. G. L. Gordon, A geometric study of the monodromy of complex analytic surfaces, Invent. Math. 1 (1977), 11-35.

6. __ On the degeneracy of a spectral sequence associated to normal crossings, Pacific J. Math. (to appear).

7. P. A. Griffiths and W. Schmid, Recent developments in Hodge theory: A discussion of techniques and results, Discrete Subgroups of Lie Groups and Application to Moduli (Internat. Colloq. Bombay, 1973), Oxford Univ. Press, Bombay, 1975, pp. 31-137.

8. H. Hironaka, Bimeromorphic maps, mimeographed notes, Warwick, 1971.

9. G. Kempf, F. Knudson, D. Mumford and B. Saint-Donat, Toroidal embeddings. I, Lecture Notes in Math., vol. 339, Springer-Verlag, New York, 1973.

10. K. Kodaira, On the structures of compact complex analytic surfaces. III, Amer. J. Math. 90 (1968), 55-83.

11. B. Malgrange, Letter to the editors, Invent Math. 20 (1973), 171-172.

12. M. Sebastiani and R. Thom, Un résultat sur la monodromie, Invent Math. 13 (1971), 90-96.

13. J. Steenbrink, Limits of Hodge structures, Invent. Math. 31 (1976), 229-257.

14. Mixed Hodge structure on the vanishing cohomology, Nordic Summer School Sympos. in Math., Oslo, 1976, pp. 525-563.

Department of Mathematics, De Paul University, Chicago, Illinois 60614 